\title{
ESTUDO DE SOLUÇÕES VPN SITE-TO-SITE SEGUNDO AS TÉCNICAS CRIPTOGRÁFICAS EMPREGADAS
}

\author{
André Luiz L. Andrade, Pedro Burached de Oliveira, Francisco Lopes de Caldas Filho, \\ Ugo Silva Dias, Rafael Timóteo de Sousa Júnior e Robson de Oliveira Albuquerque \\ Universidade de Brasília - Departamento de Engenharia Elétrica, Brasília - DF, Brasil - Zipcode 70910-900
}

\begin{abstract}
Currently, methods to protect information in networks have become essential for applications that target sensitive data traffic. For this, Virtual Private Networks (VPNs) play a key role in securing attributes such as confidentiality, authenticity, and integrity of the data packets that are submitted to the worldwide network. This paper presents a study of some characteristics of 3 opensource VPN softwares (StrongSwan, OpenVPN and Wireguard) in order to make a fair comparison between the cryptographic techniques employed by them in site-to-site tunnels.
\end{abstract}

\section{PALAVRAS-CHAVE}

Virtual Private Network (VPN), Desempenho, Jitter, Overhead de Payload, RTT

\section{INTRODUÇÃO}

Nos dias atuais, cada vez mais se faz necessário às atividades humanas a troca de informações pela internet, de maneira que a criação de métodos que visam a segurança dos dados trafegados pela rede mundial se tornam gradativamente mais importantes.

Segundo (Heyman, 2007) as conexões de rede privada virtual (VPN) estabelecem conexões seguras entre um usuário remoto e uma rede doméstica criptografando pacotes enviados pela Internet em vez de criar uma verdadeira rede privada. Sendo assim, pode-se afirmar que a utilização de VPNs tem colaborado diretamente no estabelecimento de conexões seguras entre redes, sem a necessidade de construção de infraestrutura adicional complexa, uma vez que, dependendo do cenário de uso, pode se tornar extremamente caro e problemática dependendo das características das redes comunicantes.

Existem diversas opções de softwares que permitem a construção de VPNs disponíveis no mercado, e a escolha correta de qual utilizar se baseia em fatores técnicos. Entre tais fatores, podem ser citados o incremento no consumo de CPU e memória nos concentradores VPN, aumento do RTT e jitter na comunicação, além do incremento no tamanho dos pacotes. Estes e outros fatores precisam ser levados em consideração para que seja definido qual aplicação se adequa melhor ao cenário de rede proposto.

Nesse sentido, este artigo propõe analisar características técnicas, além de comparar a performance de jitter, RTT e overhead de payload de 3 softwares opensource de VPN: StrongSwan, OpenVPN e Wireguard inseridos em um ambiente de topologia site-to-site.

Este artigo está organizado conforme se segue. Na Seção 2 são apresentados alguns trabalhos relacionados ao tema do artigo, na Seção 3 descreve algumas características e software importantes para o contexto do trabalho. A Seção 4 apresenta os cenários de testes e os respectivos resultados com algumas discussões técnicas. A Seção 5 finaliza o artigo com as conclusões e possíveis trabalhos futuros.

\section{TRABALHOS RELACIONADOS}

Com o objetivo de entender e levantar novas possibilidades para o objeto de estudo abordado neste artigo, foram revisados alguns trabalhos que possuem finalidades comuns aos que este apresenta. 
O primeiro (PUDELKO, 2018) compara as mesmas soluções opensource de VPN em uma topologia site-to-site, também abordado neste documento. Entretanto, o autor opta por avaliar outras métricas que não incluem jitter, RTT e overhead de payload, além de realizar seus experimentos por meio de uma simulação em hardware, enquanto neste trabalho, todas as simulações foram implementadas em ambiente virtual e executadas em software. Assim como neste trabalho, nos testes realizados por Pudelko, foi utilizado tráfego UDP em cima do protocolo IPv4, tendo em vista que este tipo de fluxo não depende de nenhum tipo de feedback da outra ponta comunicante.

Seguindo o mesmo padrão, o trabalho (Aleksandra e Savić, 2016) aborda apenas o software StrongSwan e compara o desempenho da taxa de transferência entre os algoritmos de criptografia disponíveis pelo software. Nesse trabalho, o autor opta por utilizar OSPF como protocolo de roteamento, enquanto neste optou-se pelo estabelecimento de rotas estáticas configuradas manualmente em cada um dos componentes responsáveis pelo encaminhamento de pacotes até atingir o servidor. Como neste artigo, o IPSec foi implementado em modo túnel e não apresentou uma diferença perceptível na taxa de transferência quando os algoritmos de integridade foram revezados, o que indica uma escolha adequada do artigo em escolher somente um algoritmo de integridade e comparar apenas os algoritmos de criptografia no parâmetro ESP em seu arquivo de configuração.

O trabalho (Khanvilkar, Khokhar, 2004) aborda as mesmas métricas apresentadas neste trabalho. No entanto, utiliza-se de outros softwares para a realização de testes que não incluem o StrongSwan e o Wireguard. Além disso, os testes foram feitos em ambiente físico real, o que pode representar uma perda significativa de desempenho, dependendo do ambiente. As métricas de jitter e RTT, assim como neste trabalho, foram obtidos utilizando o software Iperf e através do próprio sistema operacional Linux pelo comando ping.

Quanto à segurança de algoritmos de criptografia e software que implementam VPNs, foi utilizada como referência, a dissertação de mestrado (WU, 2019), que destaca a segurança do novo protocolo desenvolvido pelo Wireguard com o objetivo de estabelecer túneis seguros, o que indica que o protocolo é sólido e recomendado para a finalidade que se propõe. Para fins de estudo e estabelecimento de métricas para os algoritmos de criptografia utilizados nas implementações VPN, como tamanho de chave e complexidade, foi utilizado o artigo (Jorstad and Landgrave, 1997) para mensurar a força dos algoritmos de criptografia disponibilizados pelos softwares que estão sendo comparados.

\section{CONCEITOS RELACIONADOS}

Esta seção aborda os principais conceitos teóricos a respeito dos softwares VPN escolhidos para análise deste trabalho.

\subsection{StrongSwan}

A pilha de protocolos TCP/IP sozinha não é capaz de manter os dados em segurança, o que significa que qualquer atacante usando uma ferramenta sniffer pode acessar facilmente os dados que estão transitando na rede, desde que tenha acesso de alguma forma ao meio de comunicação. Essa condição traz severas implicações à segurança dos dados transportados. Por esse motivo, o IPSec cifra os dados que se utilizam dele e é possível operá-lo, não apenas na internet, mas também em VPNs. O StrongSwan é um software VPN opensource baseado no protocolo IPSec capaz de estabelecer túneis seguros na camada 3 do modelo OSI.

O IPSec tem dois modos de operação: modo transporte e modo túnel. O modo túnel do IPSec, escolhido para as simulações deste trabalho, opera de tal forma que o pacote IP é completamente encapsulado em um novo pacote cifrado e transferido ao ponto final do túnel, onde o software VPN ativo decifra este pacote e o repassa para o dispositivo final. Esse modo de operação é considerado mais seguro, tendo em vista que o endereço IP do remetente e do destinatário estão protegidos pelo algoritmo de criptografia escolhido pelo administrador. Todavia, quando o IPSec atua em modo transporte apenas o payload do pacote é cifrado, permitindo que um eventual atacante acesse o endereço IP original do emissor e do destinatário do túnel, além de metadados intrínsecos ao cabeçalho de rede do pacote, o que, dependendo da aplicação, pode ser considerado uma falha de segurança na sua prática. 


\subsection{OpenVPN}

O OpenVPN é uma solução VPN que faz uso do mecanismo seguro TLS/SSL para autenticação e troca de chaves entre dispositivos que desejem se comunicar de forma virtual e privada (FEILNER, 2006). O software utiliza-se do mecanismo HMAC para garantir a integridade dos pacotes entregues e pode ser configurado para operar com chaves pré-compartilhadas (PSK) ou usando certificados emitidos por autoridades certificadoras, como por exemplo o X.509. O software apresenta algumas vantagens como: flexibilidade na conexão (TCP / UDP) e é compatível com a maioria dos sistemas operacionais, incluindo o Windows.

\subsection{Wireguard}

O Wireguard é uma nova solução VPN, ainda em desenvolvimento, que difere das outras soluções por ter um nível de segurança e desempenho diferentes dos demais. Um dos fatores se deve a sua escolha no uso do processo de cifra modernos, mas ainda não explorado por outras tecnologias de tunelamento (WU,2019). O software implementa um túnel de rede seguro como um kernel da interface de rede virtual do Linux que pretende substituir soluções baseadas em IPSec e TLS, como o caso do StrongSwan e do OpenVPN.

Diferentemente do processo de identificação em softwares VPN tradicionais, no Wireguard existe um mapeamento de associação entre chaves públicas e endereços IP autorizados, o que se denomina em termos técnicos por cryptokey routing. O handshake e o mecanismo de derivação de chaves incluem um hash do número da versão do protocolo, fazendo com que duas implementações diferentes constituam em duas chaves diferentes. Essas medidas mantém o software atualizado e evitam ataques de degradação, um tipo de ataque de negação de serviço (DoS) encontrados no SSL (PUDELKO, 2018).

\section{ARQUITETURA PROPOSTA}

A topologia padrão, destacada na Figura 1, representa a comunicação entre uma estação servidora e uma estação cliente. A comunicação cliente-servidor é intermediada por dois concentradores VPN e um roteador. As rotas estabelecidas no modelo são estáticas e totalmente implementadas em ambiente virtual, operando através do software Virtual Box.

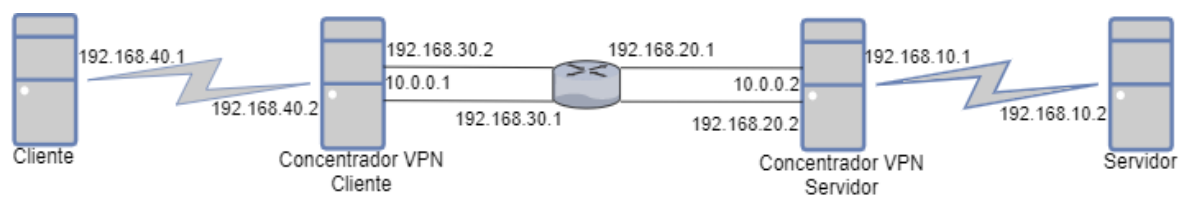

Figura 1. Topologia geral do experimento

Nas máquinas cliente e servidor, o sistema operacional usado foi o Debian 4.9.11. Foi utilizado o software Iperf para geração de tráfego UDP e o Wireshark para analisar os pacotes que passavam entre as máquinas. Os concentradores foram configurados apenas com os softwares VPN definidos na proposta de análise do trabalho, ambos com o sistema operacional Ubuntu 16.04.2. No roteador, o Wireshark foi instalado para análise do overhead do payload e observar se os pacotes que trafegam pelo túnel estão realmente cifrados, a fim de verificar a influência que os algoritmos de criptografia tem sob o desempenho da rede.

\subsection{Métricas Consideradas}

Para avaliação do comportamento das tecnologias VPN abordadas foram escolhidos 3 indicadores que serão responsáveis pela medição de desempenho das tecnologias: jitter, RTT e overhead do payload.

\subsubsection{Jitter}

De acordo com o trabalho de (HANCOCK, 2004), jitter é a variação da latência. É uma métrica de relevância principalmente para aplicações em tempo real como VoIP, jogos online e aplicações streaming em geral como 
distribuidores de conteúdo como a Netflix e Spotify. No experimento realizado, os dados relacionados à esta métrica foram obtidos através do gerador de tráfego Iperf e podem ser usados para verificar sintomas de instabilidade na rede. Em todas as 20 amostras coletadas, um único modelo de tráfego foi gerado pelo software Iperf, que consiste em um fluxo de 60 segundos de pacotes UDP carregando consigo 1000 bytes de dados.

\subsubsection{Round-Trip Time}

Segundo (KUROSE, 2013), Round Trip Time (RTT) é o tempo que leva para um pacote viajar do cliente ao servidor e de volta ao cliente. As medições de RTT foram obtidas das métricas fornecidas do sistema operacional Linux com o comando ping. Um tráfego com o protocolo ICMP de 50 pacotes foi gerado no terminal da máquina cliente e retornou o tempo médio gasto durante a viagem de todos os dados de tráfego.

\subsubsection{Overhead de Payload}

No experimento realizado, o overhead do payload será dado pela proporção do tamanho do payload dos dados enviados pelo cliente e pelo tamanho do payload de dados que passam pelo túnel e capturados pelo roteador, após o processo de cifragem. Todas as capturas levadas em consideração foram feitas usando a ferramenta tcpdump e posteriormente visualizadas no software Wireshark. Com o processo de criptografia realizado pelos concentradores de VPN, espera-se um tamanho maior dos pacotes que trafegam pelo túnel. Para esse fim, é importante medir quanto está sendo adicionado aos dados no processo de criptografia.

\subsection{Cenário I - Implementação com o Software StrongSwan}

Para os testes realizados com o StrongSwan, implementado em máquinas virtuais, foi utilizado a topologia indicada pela figura 1. O protocolo IPSec foi configurado em modo túnel, com o atributo IKE igual a aes128-sha256-modp1024, o que indica os parâmetros utilizados para as trocas de chave o algoritmo de criptografia AES-128, o algoritmo de hash SHA-256, e modp1024 como grupo Diffie-Hellman. O parâmetro ESP foi o único argumento alterado durante os testes, onde foram configurados os algoritmos AES-128, AES-256, 3DES, Blowfish e o ChaCha20 para que se fizesse o estudo comparativo proposto neste trabalho.

\subsubsection{Resultados e Análise Para o Cenário I}

Vinte amostras dos testes realizados foram coletadas e os resultados mostram que, independentemente dos algoritmos de criptografia escolhidos, o jitter apresenta uma considerável variação entre as amostras. Os algoritmos que apresentam uma média de jitter mais baixa são o Blowfish e o AES-128, enquanto algoritmos como o AES-256 mostram uma variação de RTT mais alta e podem ser vistos, em uma primeira análise, como algoritmos inadequados para serviços de streaming ou aplicações em tempo real. A Tabela 1 resume a média aritmética e mediana das amostras coletadas.

Tabela 1. Média aritmética e mediana do jitter no cenário I

\begin{tabular}{llllll}
\hline & AES 256 & AES 128 & ChaCha20 & 3DES & Blowfish \\
\hline Média & 0.1785 & 0.1233 & 0.1239 & 0.1389 & 0.1131 \\
Mediana & 0.1665 & 0.1170 & 0.1055 & 0.1335 & 0.0190 \\
\hline
\end{tabular}

Em relação ao tempo gasto de ida e volta dos pacotes ICMP observa-se, na Figura 2, que a técnica de criptografia empregada com o menor Round Trip Time foi o AES com tamanho de chave igual a 128 bits, enquanto o maior RTT foi obtida ao implementar o algoritmo de cifra de fluxo ChaCha20.

Diferente do resultado esperado, os algoritmos de cifra de bloco tiveram um desempenho de RTT melhor que o Chacha20 (cifra de fluxo). Em relação a diferença de desempenho do algoritmo AES-128 com o AES-256, julga-se ser pelo fato de o algoritmo AES-128 usar uma chave menor e, consequentemente, executar um número menor de rounds no processo de criptografia. Tendo em vista que a quantidade de dados trafegados no teste (50 pacotes ICMP) não é significativamente grande, o desempenho do ChaCha20 não supera a dos algoritmos de cifra de bloco nas circunstâncias em que os testes são feitos neste experimento

Para o caso do experimento com o StrongSwan, que implementa o protocolo IPSec, há 8 bytes a mais de payload em comparação com os outros dois softwares estudados por conta do cabeçalho da camada de transporte (UDP) fazer parte do payload da camada de rede. Os resultados de overhead de payload apresentados utilizando o software StrongSwan não indicaram diferenciação do tamanho de pacote em relação 
aos tamanhos de chave escolhido no algoritmo AES. As cifras ChaCha20, 3DES e Blowfish apresentam o mesmo overhead e mostraram-se mais otimizadas em relação ao AES nesta métrica, conforme mostra a Tabela 2.

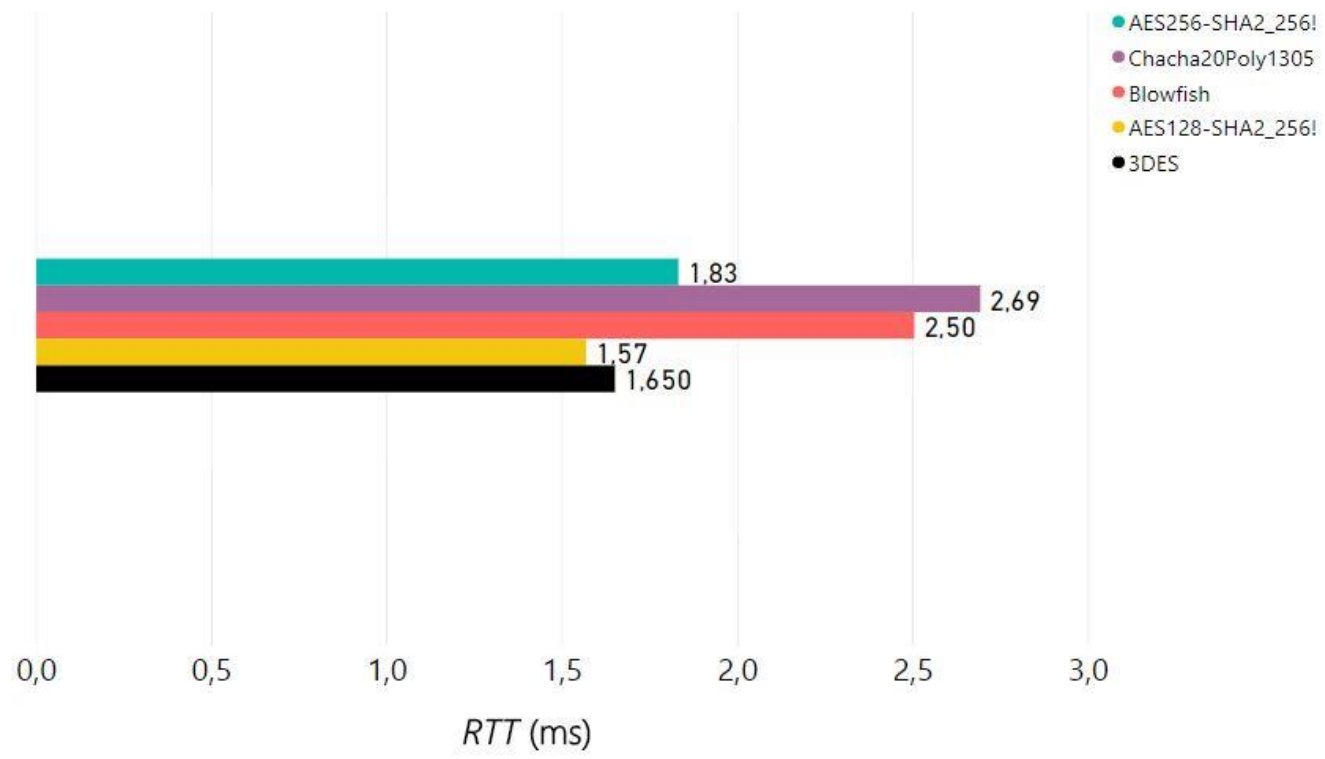

Figura 2. Média de RTT com StrongSwan Segundo as técnicas de criptografia empregadas

Tabela 2. Overhead de payload no cenário I

\begin{tabular}{llllll}
\hline & AES 256 & AES 128 & ChaCha20 & 3DES & Blowfish \\
\hline Payload Original (bytes) & 1008 & 1008 & 1008 & 1008 & 1008 \\
Payload Cifrado (bytes) & 1080 & 1080 & 1080 & 1064 & 1064 \\
Overhead (\%) & 7,14 & 7,14 & 5,55 & 5,55 & 5,55
\end{tabular}

\subsection{Cenário II - Implementação com o Software OpenVPN}

O túnel neste cenário foi configurado no modo de conexão UDP, padrão recomendado pelo software. A autenticação de pacote no Open VPN é feita, por padrão, pela função de hash SHA1 com HMAC. No cenário do experimento, optou-se pela técnica PSK para a distribuição de chaves. O parâmetro cipher do arquivo de configuração foi a única variável alterada durante os testes, onde foram utilizados os algoritmos AES-256, AES-128, DES, 3DES e Blowfish, no modo Cipher Block Chaining (CBC).

\subsubsection{Resultados e Análise do Cenário II}

Na série de 20 amostras extraídas no cenário com o OpenVPN, é observado um desempenho satisfatório em relação ao jitter quando usados os algoritmos AES-128 e DES, enquanto o 3-DES mostrou-se um algoritmo inadequado para aplicações que exigem jitter baixo. A Tabela 3 mostra os resultados de média e mediana dos testes realizados.

De acordo com a Figura 3, pode-se observar que os algoritmos AES com chaves de tamanho 128 e 256 bits apresentam baixo RTT em relação aos algoritmos DES e Blowfish. Uma observação relevante para a análise do cenário com OpenVPN é o aumento de $86 \%$ no tempo de execução com o algoritmo 3DES em relação ao 3DES implementado no cenário usando o protocolo IPSec, indicando um desempenho muito abaixo do esperado. De acordo com (DONENFELD, 2018), o atraso acima da média observado nos testes de implementação do OpenVPN pode ser justificado pelas várias cópias feitas entre o espaço do kernel e o espaço do usuário do sistema operacional dos concentradores. 
Tabela 3. Média aritmética e mediana do jitter no cenário II

\begin{tabular}{llllll}
\hline & AES 256 & AES 128 & DES & 3DES & Blowfish \\
\hline Média & 0.1897 & 0.1419 & 1,1804 & 0,2292 & 0,2267 \\
Mediana & 0,1855 & 0,1345 & 0,1890 & 0,2250 & 0,2310 \\
\hline
\end{tabular}

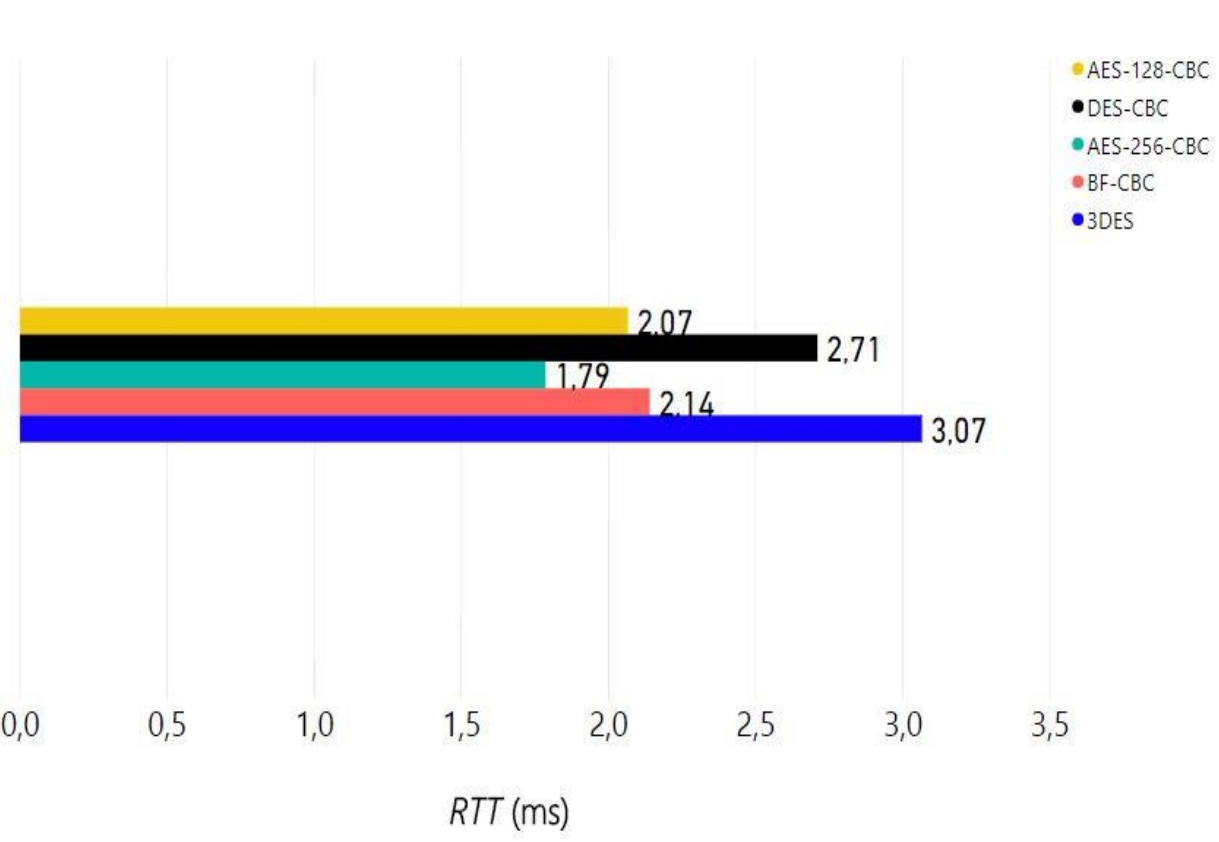

Figura 3. Média de latência com OpenVPN Segundo as técnicas de criptografia empregadas

O overhead apresentado pelo software Open VPN aponta para um aumento considerável no número de bytes acrescentados pelo processo de criptografia em relação ao StrongSwan. Também podemos ver que não há diferenciação de overhead de payload com o tamanho da chave usada no algoritmo AES, como evidenciado na Tabela 4.

Tabela 4. Overhead de Payload no cenário II

\begin{tabular}{llllll}
\hline & AES 256 & AES 128 & ChaCha20 & 3DES & Blowfish \\
\hline Payload Original (bytes) & 1000 & 1000 & 1000 & 1000 & 1000 \\
Payload Cifrado (bytes) & 1076 & 1076 & 1068 & 1068 & 1068 \\
Overhead (\%) & 7,60 & 7,60 & 6,80 & 6,80 & 6,80
\end{tabular}

\subsection{Cenário III - Implementação com o Software Wireguard}

No caso do Wireguard, não há um algoritmo de criptografia a ser comparado, tendo em vista a limitação do software para implementar algoritmos de criptografia, suportando em sua versão (0.0.20190406-wg1 xenial), testada neste trabalho, somente o algoritmo ChaCha20.

\subsubsection{Resultados e Análises para o Cenário III}

Na série de 20 amostras extraídas do experimento realizado com o software Iperf, aplicando os parâmetros de tráfego padrão indicados na Seção 4.1.1, o jitter com o Wireguard apresenta uma média de 0,1928 ms, conforme indicado na Tabela 5. É um resultado dentro do esperado em comparação com os outros softwares testados. Vale observar que foi o resultado que apresentou a menor variação nos testes realizados. Nas 20 amostras coletadas, percebe-se maior regularidade em relação à sua média, trazendo maior confiabilidade em seus resultados. A Tabela 5 mostra os resultados do jitter, média e mediana para este cenário. 
Tabela 5. Média aritmética e mediana do jitter no cenário III

\begin{tabular}{ll}
\hline & ChaCha20 \\
\hline Média & 0.1928 \\
Mediana & 0,1905 \\
\hline
\end{tabular}

O Wireguard obteve o resultado de 2,04 ms de RTT. O que pode ser considerado um resultado relativamente satisfatório em comparação com outros softwares testados. Deve-se reconhecer que, pelos testes realizados em um ambiente virtual, há uma influência relevante da limitação da CPU usada nos processos em execução nas máquinas, o que coopera para um desempenho limitado do Wireguard em relação ao IPSec implementando cifras como 3DES, AES-128 e AES-256.

O overhead de payload apresentado pelo Wireguard e pelo Open VPN demonstram que mais bytes foram acrescentados ao payload do que nos testes realizados com o IPSec, conforme mostrado na Tabela 6.

Tabela 6. Overhead de payload no cenário III

\begin{tabular}{ll}
\hline & ChaCha20 \\
\hline Payload Original (bytes) & 1000 \\
Payload Cifrado (bytes) & 1072 \\
Overhead (\%) & 7,20
\end{tabular}

\subsection{Comparação entre os Resultados}

Como forma de avaliar os resultados finais, a tabela 7 apresenta um resumo geral de cada teste e de cada ferramenta, com seu respectivo resultado, segundo os parâmetros avaliados.

Tabela 7. Comparação geral entre os resultados obtidos

\begin{tabular}{|c|c|c|c|c|c|c|c|c|}
\hline \multirow[b]{3}{*}{ Camada } & \multirow[b]{3}{*}{ VPN } & \multirow[b]{3}{*}{ Métrica } & \multicolumn{6}{|c|}{ Algoritmo Criptográfico } \\
\hline & & & \multicolumn{5}{|c|}{ Bloco } & \multirow{2}{*}{$\begin{array}{c}\text { Fluxo } \\
\text { CHACHA20 }\end{array}$} \\
\hline & & & DES & 3DES & AES 128 & AES 256 & BLOWFISH & \\
\hline \multirow{3}{*}{ IP } & \multirow{3}{*}{$\begin{array}{c}\text { STRONGSWAN } \\
\text { (IPSEC) }\end{array}$} & Jitter (ms) & - & 0,1297 & 0,1315 & 0,1855 & 0,139 & 0,1325 \\
\hline & & Latency (ms) & - & 1,65 & 1,57 & 1,83 & 2,50 & 2,69 \\
\hline & & $\begin{array}{r}\text { Overhead } \\
\text { payload (\%) }\end{array}$ & - & 5,55 & 7,14 & 7,14 & 5,55 & 5,55 \\
\hline \multirow{6}{*}{ Transporte } & \multirow{3}{*}{ OPENVPN } & Jitter (ms) & 0,1820 & 0,2448 & 0,1764 & 0,1991 & 0,2667 & - \\
\hline & & Latency $(\mathrm{ms})$ & 2,71 & 3,07 & 2,07 & 1,79 & 2,14 & - \\
\hline & & $\begin{array}{r}\text { Overhead } \\
\text { payload (\%) }\end{array}$ & 6,80 & 6,80 & 7,60 & 7,60 & 6,80 & - \\
\hline & \multirow{3}{*}{ WIREGUARD } & Jitter (ms) & - & - & - & - & - & 0,1919 \\
\hline & & Latency $(\mathrm{ms})$ & - & - & - & - & - & 2,04 \\
\hline & & $\begin{array}{r}\text { Overhead } \\
\text { payload (\%) }\end{array}$ & - & - & - & - & - & 7,20 \\
\hline
\end{tabular}

\section{CONCLUSÃO E TRABALHOS FUTUROS}

Neste trabalho foram abordadas 3 tecnologias opensource que implementam VPNs segundo as técnicas criptográficas empregadas, com o objetivo de evidenciar seu desempenho e limitações de acordo com as métricas escolhidas. Um fato que deve ser levado em consideração é a limitação computacional de processamento e memória, intrínsecas ao ambiente utilizado composto por máquinas virtuais. Entende-se que existe a possibilidade para novos testes com implementações em sistemas reais com recursos computacionais mais robustos, tendo em vista que espera-se que quanto maior a capacidade computacional de um sistema, mais sensível os resultados são em relação aos algoritmos de criptografia implementados, devido a velocidade de processamento dos mesmos. 
Com relação a estudos de segurança, com o avanço cada vez maior da capacidade computacional, pode-se esperar que caso um atacante tenha conhecimento acerca das métricas de overhead de payload dos algoritmos de criptografia, usando por exemplo, métodos de inteligência artificial, é possível analisar este dado e inferir qual algoritmo poderia estar sendo utilizado no túnel. Isto é uma hipótese que necessita de estudos mais aprofundados, mas entende-se que é possível realizar esta análise como um dos parâmetros para se estimar um algoritmo de criptografia empregado na comunicação cifrada.

\section{AGRADECIMENTO}

Os autores agradecem o apoio das Agências brasileiras de pesquisa, desenvolvimento e inovação CNPq (Projeto INCT em Segurança Cibernética 465741/2014-2), CAPES (Projeto FORTE 23038.007604/2014-69 e PROBRAL 88887.144009/2017-00) e Fundação de Apoio à Pesquisa do Distrito Federal FAPDF (Projetos UIoT 0193.001366/2016 e SSDDC 0193.001365/2016), bem como do Gabinete de Segurança Institucional da Presidência da República (TED 002/2017) e do Laboratório LATITUDE/UnB (Projeto SDN 23106.099441/2016-43).

\section{REFERÊNCIAS}

Christof Paar e Jan Pelzl, 2009 “Understanding Cryptography”. Published by Springer. Bochum, Germany.

Eric Crist and Jan Just Keijser, 2015 "Mastering OpenVPN", Packt Publishing, ISBN: 978-1-78355-313-6.

Francisco L. de Caldas Filho, Rafael T. de Sousa Júnior, Lucas M. C. e Martins, Ingrid Palma Araújo, Fábio L. L. de Mendonça and João Paulo C. L. da Costa, 2017 "Design and Evaluation of a Semantic Gateway Prototype for IoT Networks", University of Brasília.

Heyman, Karen, 2007 "A new virtual private network for today's mobile world." Computer 40.12 (2007): 17-19.

J. Hancock, 2004 "Jitter - understanding it, measuring it, eliminating it part 1: Jitter fundamentals," High Frequency Electronics, pp. 44-50.

Jason A. Donenfeld, 2018 "WireGuard: Next Generation Kernel Network Tunnel”.

Kurose, James F and Ross, Keith W, 2013 “Computer Networking: A Top Down Approach” Boston, MA, USA.

Markus Feilner, 2006 "OpenVPN Building and Integrating Virtual Private Networks", Packt Publishing,Birmingham, B27 6PA, UK.

Maximilian Pudelko, 2018 "Performance Analysis of VPN Gateways", Department of Informatics, Technical University of Munich.

Norman D. Jorstad and T. S. Landgrave, 1997 "Cryptographic algorithm metrics", 20th National Information Systems Security Conference, Baltimore, MD.

Peter Wu, 2019 "Analysis of the WireGuard protocol", Eindhoven University of Technology, Department of Mathematics and Computer Science.

Redžovic, Hasan and Smiljani 'c, Aleksandra and Savi 'c, Bogdan, 2016"Performance evaluation of Software Routers with VPN features" ' 24th Telecommunications Forum (TELFOR), pages: 1-4, IEEE, 2016.

Shashank Khanvilkar and Ashfaq Khokhar, 2004 "Virtual Private Networks: An Overview with Performance Evaluation", University of Illinois at Chicago, 2004 IEEE, IEEE Communications Magazine - pages 146-154. 